\title{
Performance Simulations of WLAN and Zigbee in Electricity Substation Impulsive Noise Environments
}

\author{
S A Bhatti, Q Shan, R Atkinson and I A Glover \\ Department of Electronic \& Electrical Engineering, University of Strathclyde \\ 204 George Street, Glasgow G1 1XW, UK \\ Email: shahzad.bhatti@strath.ac.uk
}

\begin{abstract}
A physical layer performance evaluation of WLAN (IEEE 802.11b) and Zigbee (IEEE 802.15.4) receivers in the presence of impulsive noise is presented. Broadband impulsive noise is modeled as a Symmetric Alpha-Stable process. The parameters of the impulsive noise model are estimated from data recorded from an electricity transmission substation (ETS). The results show that the performance degradation of the Zigbee receiver is small whilst the WLAN receiver may suffer more significant degradation.
\end{abstract}

Index Terms - impulsive noise; non-Gaussian noise; WLAN; Zigbee; symmetric alpha-stable process; electricity substation.

\section{INTRODUCTION}

The traditional power transmission paradigm is changing. The more efficient, complex, electricity grid of the future incorporating distributed generation using renewable energy sources, a disparate array of energy storage technologies and active collaboration of consumers in load balancing via smart metering and demand-side management will require practically continuous grid monitoring and instant grid control. Wireless technologies represent a convenient means of achieving the necessary communications connectivity in substations with significant flexibility and cost advantages over fiber and copper cabling. Wireless local area network (WLAN) technology is used extensively for networking computers with peripheral devices, and is an obvious candidate for deployment in substations. Zigbee technology, based on the IEEE 802.15.4 standard, is another popular short range technology which has been used in many commercially available wireless sensor networks (WSN) solutions [1]. The proposed development of the smart grid and its evolution from the existing grid structure has been a topic of intense research for a decade. All proposals, at their highest level, center on the integration of the power transmission and communications networks. Whilst wireless networks have obvious benefits of cost and flexibility over wired networks, concerns remain which need to be addressed. One such concern is the practical performance that can be expected of commercially available wireless technologies in the unusual and challenging noise environment of electricity substations. This noise environment may be intensely impulsive in character due to partial discharge, power electronic switching and other transient processes [2]. The degree to which a dominantly impulsive noise environment might degrade the performance of wireless technologies, primarily designed to operate in a Gaussian noise environment, is an important consideration.

The rest of the paper is organized as follows. Sections-II details the measurement campaign; the data recorded from this campaign is used to estimate the parameters of impulsive noise model. Section-III gives an account of impulsive noise modeling and details of the Symmetric $\alpha$-Stable $(\mathrm{S} \alpha \mathrm{S})$ noise model which is employed to model the electricity transmission substation noise environment. It also explains the process of estimation of model parameters. Section-IV describes the physical layer specifications of the WLAN and Zigbee receivers. Section-V consists of validation results of the physical layer simulations of WLAN and Zigbee and their performance evaluation in the presence of broadband impulsive noise. A summary of findings is provided in Section-VI.

\section{MEASUREMENTS}

The impulsive noise detection system (INDS) is comprised of four broadband antennas, a high bandwidth digital storage oscilloscope (DSO) and a 1TB external hard drive (HDD). This system was deployed in control room of Strathaven $400 / 275 / 132 \mathrm{kV}$ air-insulated electricity substation in UK, owned by Scottish Power Limited. A specification of the INDS is given in Table 1. Further details of the INDS were reported in [1].

TABLE 1 SPECIFICATION OF INDS

\begin{tabular}{|c|c|}
\hline & Antennas \\
\hline Type & Frequency Range \\
\hline Low-band TEM half-horn & $716 \mathrm{MHz}-1.98 \mathrm{GHz}$ \\
\hline High-band TEM half horn & $1.905 \mathrm{GHz}-5.1 \mathrm{GHz}$ \\
\hline Disk-cone & $10 \mathrm{MHz}-1 \mathrm{GHz}$ \\
\hline WLAN dual-band & $1.4 \& 5 \mathrm{GHz}$ (WLAN Bands) \\
\hline \multicolumn{2}{|c|}{ Detection and Recording Equipment } \\
\hline $\begin{array}{l}\text { High-bandwidth DSO } \\
\text { External HDD }\end{array}$ & $\begin{array}{l}\text { Bandwidth : } 6 \mathrm{GHz} \\
\text { Storage Capacity: } 1 \mathrm{~TB}\end{array}$ \\
\hline
\end{tabular}

The recorded spectra show that multiple sources of coherent interference are present from terrestrial broadcasting and satellite communication systems [4]. To extract impulsive events (which are of particular interest here), two stages of data processing are employed. First, a de-noising algorithm, based on the Wavelet Packet Transformations (WPT), was employed and second a basic impulse feature extraction algorithm was 
applied. Details of the data processing can be found in [4-5]. The assumption that the impulsive process so extracted is qualitatively characteristic of impulsive noise in other substations or at other locations within the measurement substation remains to be tested. There is no assumption, however, that the measurements are quantitatively characteristic of impulsive noise intensity since the performance of the technologies tested is characterized as a function of signal to impulsive noise ratio and compared with that for white Gaussian noise.

\section{IMPULSIVE NOISE MODEL}

Impulsive noise process is considered to be result of a large number of noise sources. Its characteristics are dependent on the spatial and temporal distributions of the individual noise sources and their propagation to receiver. The noise which is received at the receiver front-end is superposition of narrow pulses produced by individual noise sources. Middleton used filtered-impulse mechanism in the development of nonGaussian impulsive noise models which considers the observations regarding spatial and temporal distribution and propagation to the receiver [6]. Nikias and Shao have used the same mechanism in the development of stable impulsive noise model [8]. Gaussian mixture distributions, Middleton's models and $\mathrm{S} \alpha \mathrm{S}$ processes are among the popular, heavy-tailed, distributions, used to model impulsive noise. Here, 'heavy-tail' means that their probability density function (pdf) tails decay at a rate less than the Gaussian pdf. Impulsive noise can be divided into two types based on the relative bandwidth of noise and receiver [6]. Middleton's class A model is appropriate for the narrowband noise (i.e. when the noise spectrum is narrow than receiver bandwidth). The evaluation of WLAN performance in the presence of Middleton Class A noise is addressed in [5]. Here, $\mathrm{S} \alpha \mathrm{S}$ distributions are used to model broadband impulsive noise (i.e. noise with a spectrum that is wider than the receiver bandwidth). The $\mathrm{S} \alpha \mathrm{S}$ distribution parameters of the impulsive noise model are estimated using the measured data.

The $\mathrm{S} \alpha \mathrm{S}$ distribution $\left[\exp \left(-|w|^{\alpha}\right)\right]$, is a generalization, of the Gaussian characteristic function i.e. $\exp \left(-w^{2}\right)$. The $\mathrm{S} \alpha \mathrm{S}$ distribution reduces to Gaussian for $\alpha=2$. In addition to Gaussian case, closed form solution exists for only one value of $\alpha$ ( $\alpha=1$; which is Cauchy distribution). The $\mathrm{S} \alpha \mathrm{S}$ model is a good, and relatively simple, approximation to the Middleton Class B model, i.e. pdf of an $\mathrm{S} \alpha \mathrm{S}$ is a close approximation to the pdf of class B noise [8]. The Middleton Class B model is appropriate for broadband impulsive noise [6]. The relationship between Class B noise and the S $\alpha \mathrm{S}$ process is established via their characteristic functions. The characteristic function of an $\mathrm{S} \alpha \mathrm{S}$ process is given by:

$$
\Phi(\omega)=e^{j \delta \omega-\gamma|\omega|^{\alpha}}
$$

where $\alpha(0<\alpha \leqslant 2)$ is the characteristic exponent which determines the shape of the distribution. $\delta$ is location parameter and $\gamma$ is the dispersion of the distribution (describing the spread of the distribution around $\delta$ ). For $\alpha$ in the range $\{1,2\}, \delta$ can be identified as the distribution mean and for $\alpha$ in the range $\{0,1\}$, it can be identified as the distribution median. As there is no general closed form expression for the S $\alpha \mathrm{S}$ pdf, power series expansion, derived in [9], is employed in this work. Fig. 1 shows the pdf of an S $\alpha \mathrm{S}$ impulsive noise process which is close to Gaussian near zero but decays more slowly than Gaussian in the tails. Gaussian tails are exponential but $\mathrm{S} \alpha \mathrm{S}$ tails are algebraic. Tail thickness of $\mathrm{S} \alpha \mathrm{S}$ pdf depends on the value of $\alpha$ (the smaller $\alpha$, the thicker the tails).

\section{A. Generalized SNR}

The SaS distribution does not have finite second order moments and all $\mathrm{S} \alpha \mathrm{S}$ signal processing is therefore based on fractional lower order moments (FLOMs) [10]. As a consequence, the use of a traditional signal-to-noise ratio (SNR) is not appropriate. BER performance can be characterized, however, as a function of dispersion, $\gamma$ and signal variance $s$. A generalized SNR (GSNR) [11] can be defined as:

$$
G S N R=10 \log _{10}(s / \gamma)
$$

\section{B. Parameter Estimation}

Over the last four decades the problem of $\mathrm{S} \alpha \mathrm{S}$ model parameter estimation has been addressed by many authors. A fast and efficient estimator, developed in [12], is based on asymptotic behavior of extreme-order statistics of data. It estimates all three parameters and involves relatively simple computations compared to other $\mathrm{S} \alpha \mathrm{S}$ estimators. A brief description of this estimator is given below.

Let $X_{1}, X_{2}, \ldots, X_{l}, \ldots, X_{N}$ be a measured series of independent $\mathrm{S} \alpha \mathrm{S}$ random variables with unknown values of parameters $\alpha, \gamma$ and $\delta$. The estimation procedure is as follows. $\delta$ is estimated first since it does not require knowledge of $\alpha$ or $\gamma . \alpha$ is estimated next followed by estimation of $\gamma$.

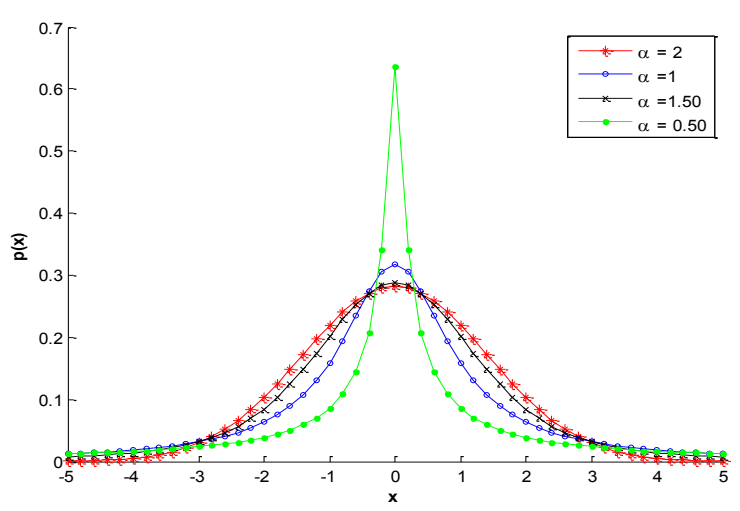

Fig. 1. S $\alpha$ S probability density functions for selected values of $\alpha$ 
1) The location parameter $\delta$ is defined as sample mean of median of the measurement series.

$$
\hat{\delta}=\operatorname{median}\left\{X_{1}, X_{2}, \ldots, X_{N}\right\}
$$

2) The characteristic exponent $\alpha$ is estimated using three steps. First, the centered data series is divided into $K$ nonoverlapping segments of equal length $L=N / K$. Second, the logarithms of the maximum and minimum values of data segment are calculated, i.e.:

$$
Y_{k, \text { max }}=\log \left(X_{k, \text { max }}\right) \text { and } Y_{k, \text { min }}=\log \left(X_{k, \text { min }}\right)
$$

Their corresponding standard deviations are given by:

$$
\begin{gathered}
s_{\max }=\sqrt{\frac{1}{K-1} \sum_{k=1}^{K}\left[Y_{k, \text { max }}-\bar{Y}_{\max }\right]^{2}} ; \bar{Y}_{\max }=\frac{1}{K} \sum_{k=1}^{K} Y_{k, \text { max }} \\
s_{\text {min }}=\sqrt{\frac{1}{K-1} \sum_{k=1}^{K}\left[Y_{k, \text { min }}-\bar{Y}_{\min }\right]^{2}} ; \bar{Y}_{\min }=\frac{1}{K} \sum_{k=1}^{K} Y_{k, \text { min }}
\end{gathered}
$$

Third, the estimated characteristic exponent $\alpha$ is given by:

$$
\hat{\alpha}=\frac{\pi}{2 \sqrt{6}}\left(\frac{1}{s_{\max }}+\frac{1}{s_{\min }}\right)
$$

The reasons for this choice of estimator are explained in [12].

3) The estimator for $\gamma$, based on the theory of fractional lower order moments of the pdf, is defined as:

$$
\hat{\gamma}=\left[\frac{\frac{1}{N} \sum_{l=1}^{N}\left|X_{l}-\hat{\delta}\right|^{p}}{C(p, \hat{\alpha})}\right]^{\frac{\hat{\alpha}}{p}}
$$

where $C(p, \hat{\alpha})$ is given by:

$$
C(p, \hat{\alpha})=\frac{\Gamma\left(1-\frac{p}{\hat{\alpha}}\right)}{\cos \left(\frac{\pi}{2}\right) \Gamma(1-p)}
$$

The recommended order of fractional moment $p$ is $\alpha / 3$ and $\Gamma$ is Gamma function in (9). The performance of these estimators has been shown to be very satisfactory [12]. They are used, in simulations, to estimate the parameters of $\mathrm{S} \alpha \mathrm{S}$ noise model, which is employed to evaluate the vulnerability WLAN and Zigbee receivers to broadband impulsive noise.

\section{WLAN AND ZIGBEE}

A brief overview of IEEE $802.11 \mathrm{~b}$ (WLAN) and IEEE 802.15.4 (Zigbee) standards is given below.

IEEE $802.11 \mathrm{~b}$ operates in the $2.4 \mathrm{GHz}$ band and variously uses direct sequence spread spectrum (DSSS) or frequency hopping spread spectrum (FHSS). It supports transmission rates of $1,2,5.5$ and $11 \mathrm{Mbit} / \mathrm{s}$ or modes 1,23 and 4 respectively. The different transmission rates are obtained by varying the modulation order or type. $1 \mathrm{Mbit} / \mathrm{s}$ is realized using differential binary phase shift keying (DBPSK) whilst $2 \mathrm{Mbit} / \mathrm{s}$ uses differential quadrature phase shift keying (DQPSK). Higher data rates of $5.5 \mathrm{Mbit} / \mathrm{s}$ and $11 \mathrm{Mbit} / \mathrm{s}$ use complimentary code keying (CCK). The equivalent complex baseband representation of a CCK signal [13] is given by:

$$
\begin{gathered}
c=\left\{e^{j\left(\varphi_{1}+\varphi_{2}+\varphi_{3}+\varphi_{4}\right)}, e^{j\left(\varphi_{1}+\varphi_{3}+\varphi_{4}\right)}, e^{j\left(\varphi_{1}+\varphi_{2}+\varphi_{4}\right)},-e^{j\left(\varphi_{1}+\varphi_{4}\right)}\right. \\
\left., e^{j\left(\varphi_{1}+\varphi_{2}+\varphi_{3}\right)}, e^{j\left(\varphi_{1}+\varphi_{3}\right)},-e^{j\left(\varphi_{1}+\varphi_{2}\right)}, e^{j \varphi_{1}}\right\}
\end{gathered}
$$

where $c=\left\{c_{0}\right.$ to $\left.c_{7}\right\}$

IEEE 802.15.4 was published in 2006 and specifies the physical (PHY) and MAC layer for a Wireless Personal Area Network (WPAN) with tight constraints on power and bandwidth. Devices compliant with this standard are power efficient and have low manufacturing costs. These devices operate in snooze mode with short wakeup time $(30 \mathrm{~ms})$ compared to competing technologies. (Bluetooth, for example has a wakeup time of 3 s.) This PHY and MAC standard is popular in low power, low data-rate, technologies. The WirelessHART protocol is an example which uses IEEE 802.15.4 for its MAC and PHY layers. It operates in three different ISM (Industrial Scientific and Medical) frequency bands. These bands [14] are specified in Table 2

TABLE 1 OPERATING FREQUENCIES

\begin{tabular}{|c|c|c|}
\hline $\begin{array}{c}\text { Frequency } \\
\text { Range } \\
\text { (MHz) }\end{array}$ & $\begin{array}{c}\text { No of } \\
\text { Channels }\end{array}$ & Region \\
\hline $868-868.6$ & 1 & Emericas and Australia \\
\hline $902-928$ & 10 & Worldwide \\
\hline $2400-2483.5$ & 16 & \\
\hline
\end{tabular}

IEEE 802.15.4 uses Direct Sequence Spread Spectrum (DSSS) to ensure low power spectral density and increased immunity to noise from nearby networks. $868 \mathrm{MHz}$ mode (mode 1) uses binary phase shift keying (BPSK) in which each symbol (in this case each symbol is one bit) is mapped to 15 chips. The $2400 \mathrm{MHz}$ mode (mode 2) uses orthogonal quadrature phase shift keying (OQPSK) with each symbol (four bits) being mapped to a 32-chip PN sequence. The spreading PN sequences are listed in the standard [14]. Receiver sensitivity is specified to be $-92 \mathrm{dBm}(868 \mathrm{MHz}$ 
mode) and $-85 \mathrm{dBm}(2400 \mathrm{MHz}$ mode) for any IEEE 802.15.4 complaint device. The transmitted power must conform to the regional power regulation standards.

\section{SIMULATIONS AND RESULTS}

\section{A. Impulsive noise model}

The impulsive noise model is based on the SaS process. The parameter estimation algorithm is described in Section III. The noise model is validated by comparison to the amplitude cumulative distribution function (ACDF) of the recorded data. The agreement between data and model is illustrated in Fig. 2. The estimated values of $\delta, \alpha$ and $\gamma$ are 1.18E-7, 1.4131 and $6.34 \mathrm{E}-7$ respectively.

\section{Validation of WLAN and Zigbee simulations}

Validation of the physical layer simulation used for IEEE 802.11 b can be found in [7]. Validation of the physical layer simulation used for IEEE 802.15.4 is presented in Fig. 3. This validation comprises BER versus $\mathrm{E}_{\mathrm{b}} / \mathrm{N}_{0}$ curves for both modes 1 and 2.

\section{3) BER performance of WLAN}

Fig. 4 shows the BER performance of the WLAN receiver (all four modes) in the presence of impulsive and Gaussian noise. The impulsive noise is generated using the broadband $\mathrm{S} \alpha \mathrm{S}$ noise model. BER is plotted against GSNR (which reduces to half (as a ratio, not decibels) of the SNR for Gaussian noise). It is evident from the results that the performance of the WLAN receiver is degraded by approximately $11 \mathrm{~dB}$ for a BER of $10^{-3}$. It seems likely from other work [11], that a Cauchy receiver would perform better than the matched filter receiver in a broadband impulsive noise environment. Further work, however, is needed to make a definitive statement about this.

The performance of the WLAN receiver in the presence of narrowband impulsive noise is presented in [7]. Its degrading effect on receiver performance with respect to an equivalent amount of Gaussian noise is much less than that caused by broadband impulsive noise and, for practical engineering purposes, can probably be neglected.

\section{4) BER Performance of Zigbee}

Fig. 5 shows the BER performance of a Zigbee receiver in the presence of impulsive and Gaussian noise. The performance degradation due to impulsive noise is of the order of $2 \mathrm{~dB}$ worse than that due to Gaussian noise. This is much less than the corresponding degradation in WLAN receiver performance. Zigbee supports a much lower maximum datarate $(250 \mathrm{kbps})$, however, than that $(11 \mathrm{Mbps})$ of mode 4 of IEEE 80211(b).

\section{CONCLUSIONS}

Broadband impulsive noise has been modeled using an $\mathrm{S} \alpha \mathrm{S}$ process and the parameters of the model have been estimated from data recorded in a $400 \mathrm{kV}$ electricity transmission substation. Simulations have been used to evaluate the expected bit-error-ratio performance of WLAN and Zigbee receivers in the presence of the modeled noise process providing insight into the possible performance of these technologies when deployed in electricity substations. The performance of both technologies is degraded when operating in the impulsive noise environment compared to their performance in a Gaussian noise environment with equivalent noise intensity. The degradation in the performance of Zigbee technology is modest whilst the degradation for WLAN technology is more severe. The difference in the performance degradation of WLAN and Zigbee is probably because of the difference in process gain of spreading in both technologies.

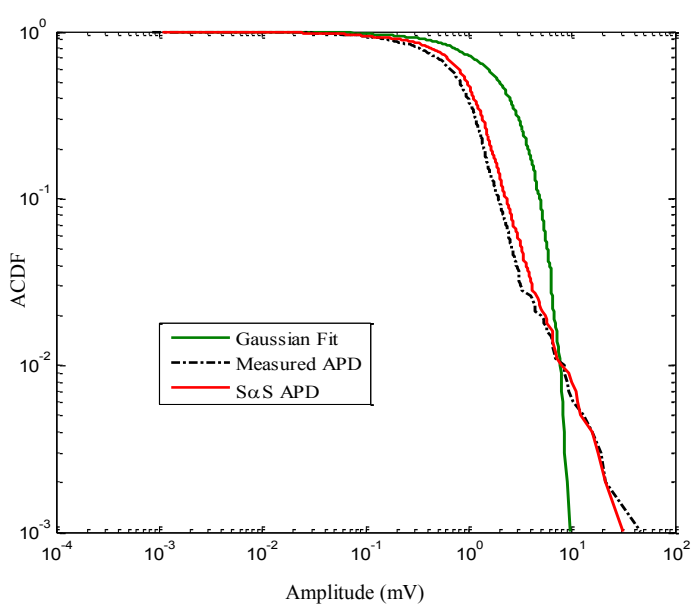

Fig. 2. Amplitude cumulative distribution of recorded data compared with best-fit Gaussian and $\mathrm{S} \alpha \mathrm{S}(\alpha=1.41)$ distributions

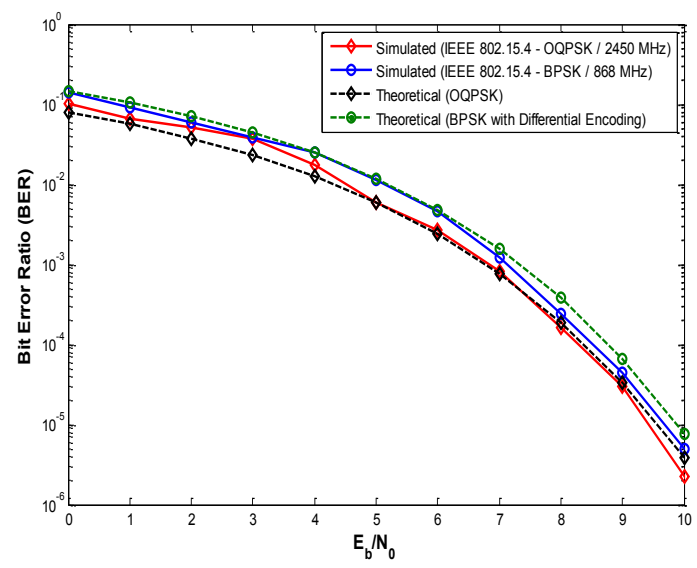

Fig. 3 Validation of the Zigbee (IEEE 802.15.4) physical layer simulation 


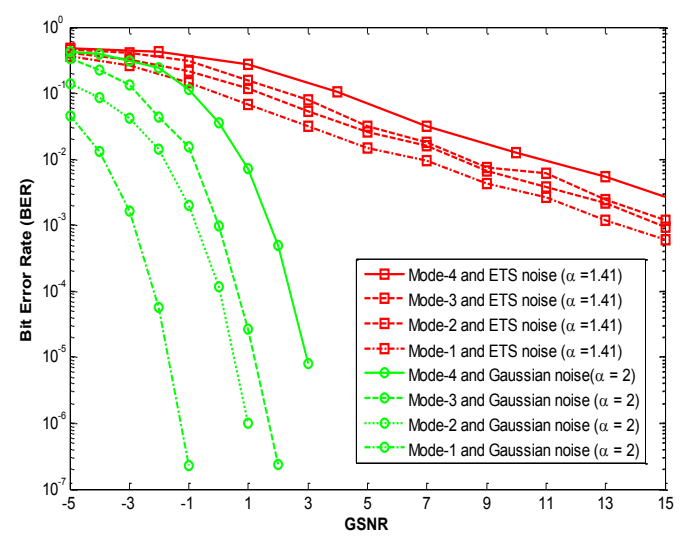

Fig. 4. BER performance of WLAN (IEEE 802.11b) receiver in the presence of electricity transmission substation (ETS) impulsive noise and Gaussian noise.

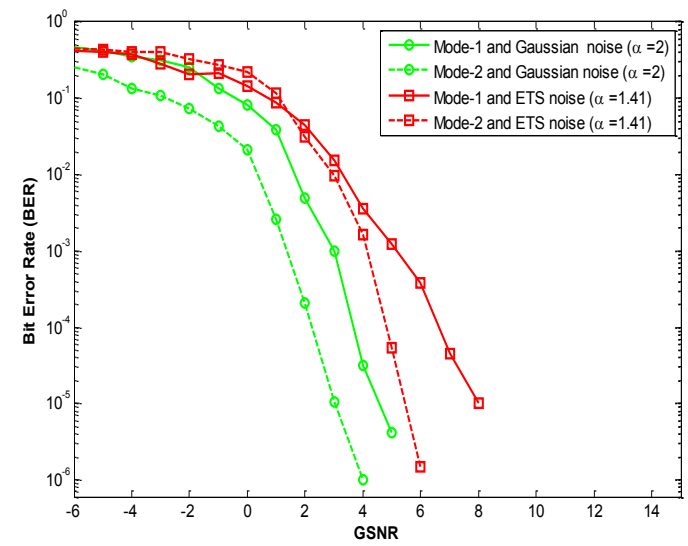

Fig. 5. BER performance of Zigbee (802.15.4) in the presence of electricity transmission substation (ETS) impulsive noise and Gaussian noise

\section{ACKNOWLEDGMENT}

The authors thank the UK Engineering and Physical Sciences Research Council (EPSRC) and University of Engineering and Technology (UET) Taxila, Pakistan for financial support of this work.

\section{REFERENCES}

[1] Y. Ke, G. Runquan, Z. Cuixia, C. Minhui, L. Ruiqiang, and W. Jiaxin, "ZigBee-Based Wireless Sensor Networks," in
International Forum on Information Technology and Applications, 2009, vol. 1, pp. 618-621.

[2] G. Madi et al., "Impacts of impulsive noise from partial discharges on wireless systems performance: application to MIMO precoders," EURASIP Journal on Wireless Communications and Networking, vol. 2011, no. 1, p. 186, Nov. 2011.

[3] Q. Shan, I. A. Glover, P. J. Moore, I. E. Portugues, M. Judd, R. Rutherford, and R. J. Watson, "TEM Horn Antenna for Detection of Impulsive Noise," in EMC Europe 2008, Hamburg, Germany, 2008.

[4] Q. Shan, S. Bhatti, I. A. Glover, R. Atkinson, I. E. Portugues, P. J. Moore, and R. Rutherford, "Extraction of Impulsive Noise from Measurements in a $400 \mathrm{kV}$ Electricity Substation," Proceedings of the $4^{\text {th }}$ IASME/WSEAS International Conference on Energy and Environment, Cambridge, UK, 2009, pp. 135139.

[5] Shan Q, SA Bhatti, I A Glover, Atkinson R, Moore P J, Portugues I E, R Rutherford, "Characteristics of impulsive noise in electricity substations", European Signal Processing Conference (EURSIP 2009), Glasgow, UK

[6] D. Middleton, "Non-Gaussian Noise Models in Signal Processing for Telecommunications: New methods and results for Class A and Class B noise models", IEEE Transactions on Information Theory, vol. 45, no. 4, pp. 1129-1149, May 1999.

[7] S A Bhatti, Shan Q, I A Glover, Atkinson R, Moore P J, Portugues I E, R Rutherford: "Impulsive Noise Modelling and Prediction of its Impact on the Performance of WLAN Receiver", $17^{\text {th }}$ European Signal Processing Conference(EUSIPCO 09), Glasgow, UK, August 2009

[8] C. L. Nikias and M. Shao, "Signal Processing with Alpha-Stable Distributions and Applications", New York: Wiley, 1995.

[9] Y. Kim, G.T. Zhou, "Representation of the Middleton Class-B model by symmetric alpha-stable processes and chidistributions", Proceedings of the 4th International Conference on Signal Processing, ICSP'98, Vol. 1, October 1998, pp. 180183.

[10] M. Shao and C. L. Nikias, "Signal processing with fractional lower order moments: stable processes and their applications," Proceedings of the IEEE, vol. 81, pp. 986-1010, 1993.

[11] Adler,R., Feldman, R.,Taqqu, M.,(editors), A Practical Guide to Heavy Tails: Statistical Techniques and Applications, Birkhauser, 1998

[12] G. A. Tsihrintzis and C. L. Nikias, "Fast estimation of the parameters of alpha-stable impulsive interference", IEEE Transactions on Signal Processing, vol. 44, no 6, pp. 14921503, June 1996.

[13] IEEE Std. 802.11b-1999 Edition (R2003), Part11: Wireless LAN Medium Access Control (MAC) and Physical Layer (PHY) specifications: Higher-Speed Physical Layer Extension in the $2.4 \mathrm{GHz}$ Band

[14] IEEE Std 802.15.4-2006, Part 15.4: Wireless Medium Access Control (MAC) and Physical Layer (PHY) Specifications for Low-Rate Wireless Personal Area Networks (WPANs), 2006. 